\title{
FAR INFRARED REFLECTANCE OF A MIXED PHASE Y-Ba-Cu-O SUPERCONDUCTOR
}

\author{
K. F. Renk, H. Lengfellner, P. E. Obermayer, \\ W. Ose, H. H. Otto, and T. Zetterer \\ Institut für Angewandte Physik, Universität Regensburg \\ 8400 Regensburg, FRG \\ and \\ W. Schindler and G. Saemann-Ischenko \\ Physikalisches Institut, Universität Erlangen \\ 8520 Erlangen, FRG
}

Received September 21, 1987

We have studied the far infrared reflectance of the mixed phase superconductor $\mathrm{Y}_{1.2} \mathrm{Ba}_{0.8} \mathrm{CuO}_{4-y}$. The reflectance spectrum shows sharp phonon resonances and an electronic contribution that we attribute to reflection at a mesh-like structure of the conducting phase embedded in nonconducting material; in comparison, the reflectance of single phase superconducting material is mainly determined by the dynamical electronic conductivity.

Key word: High $T_{c}$ Superconductivity

After the discovery of high temperature superconductivity ${ }^{1,2}$ far infrared studies have been used for characterization of properties of the new superconducting materials; studies have been performed for $\mathrm{La}-\mathrm{Ba}-\mathrm{Cu}-\mathrm{O}^{3}$. $\mathrm{La}-\mathrm{Sr}-\mathrm{Cu}-\mathrm{O}^{3-7}$ and also for $\mathrm{YBa}_{2} \mathrm{Cu}_{3} \mathrm{O}_{7}$ compounds. ${ }^{8-1}$ I Recently a transmission study for a mixed phase powder sample of $\mathrm{Y}_{1.2} \mathrm{Ba}_{0.8} \mathrm{CuO}_{4-y}$ has been reported. ${ }^{14}$ In this paper we present reflection measurements for a mixed phase $\mathrm{Y}_{1.2} \mathrm{Ba}_{0.8} \mathrm{CuO}_{4-\mathrm{Y}}$ sample and compare the results with reflection measurements for a single phase $\mathrm{YBa}_{2} \mathrm{Cu}_{3} \mathrm{O}_{7}$ sample.

We prepared samples from appropriate amounts of $\mathrm{Y}_{2} \mathrm{O}_{3}, \mathrm{BaCO}_{3}$ and $\mathrm{CuO}$. The powders were carefully 
mixed, pressed to pellets (diameter $12 \mathrm{~mm}$ ) and sintered in oxygen at $950^{\circ} \mathrm{C}$ for twelve hours. The mixed phase (dark green) sample had a specific resistance $(0.2 \Omega \mathrm{cm}$ ) that remained almost constant up to the superconducting transition at $88 \mathrm{~K}$ (inset of Fig. 1a); the width of the transition was about $7 \mathrm{~K}$ and the sample became fully superconducting at $75 \mathrm{~K}$. For comparision, we prepared a single phase (black) $\mathrm{YBa}_{2} \mathrm{Cu}_{3} \mathrm{O}_{7}$ sample. This sample had a specific resistance that decreased (from $10^{-3} \Omega \mathrm{cm}$ at $300 \mathrm{~K}$ ) with decreasing temperature and had a sharp superconducting transition at $90 \mathrm{~K}$ (inset of Fig. 1b). X-ray studies, performed for further characterization, showed that the two systems which we studied had characteristic properties of mixed phase and single phase superconductors, respectively. ${ }^{12.13}$ While the single phase superconductor

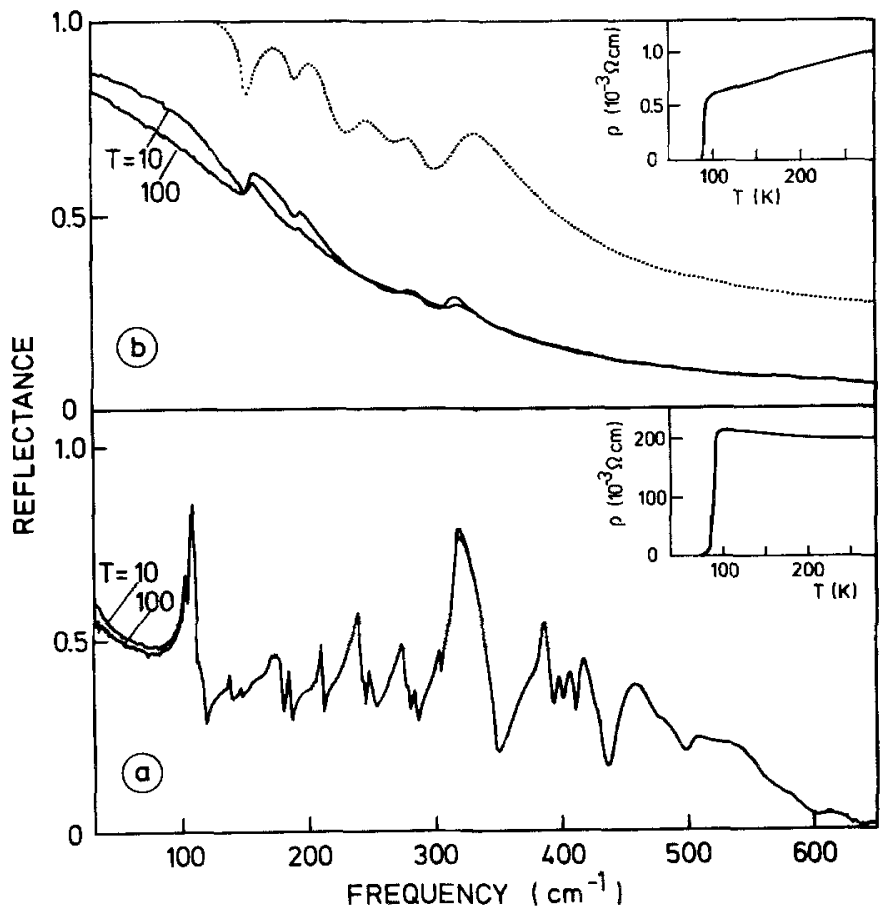

Figure 1. Far infrared reflectance of the mixed phase superconductor $\mathrm{Y}_{1.2} \mathrm{Ba}_{0.8} \mathrm{CuO}_{4-\mathrm{y}}(\mathrm{a})$ and a single phase superconductor $\mathrm{YBa}_{2} \mathrm{CuO}_{7}$ (b) together with a calculated reflectance curve ${ }^{8}$; insets, specific resistances. 
(at $77 \mathrm{~K}$ ) showed levitation in a magnetic field, the mixed phase superconductor did not. Visual inspection with a microscope showed that the sample had a surface consisting of black and green regions, with more green than black material. This picture is consistent with a ratio of about 4:1 of $\mathrm{Y}_{2} \mathrm{BaCuO}_{5}$ and $\mathrm{YBa}_{2} \mathrm{Cu}_{3} \mathrm{O}_{7}$. The shape of the regions was very irregular, a typical distance betweyen different black regions was of the order of few $10^{-3} \mathrm{~cm}$. The far infrared reflectance was deterpined by use of a Fourier spectrometer (resolution $2 \mathrm{~cm}^{-1}$ ). The reflectance was obtained by comparing the sample reflectivity with the reflectivity of an aluminium mirror: the sample surface was rough and untreated.

Experimental results are shown in Fig. 1a for a mixed phase sample and in Fig. 1b for a single phase sample. The reflectance spectrum of the mixed phase sample has a complicated structure, with characteristic Reststrahlen features in the whole frequency region covered by our study. There are two strong narrow resonances f near $102 \mathrm{~cm}^{-1}$, and a strong broad resonance (at $317 \mathrm{~cm}^{-1}$ ) and further structures. The halfwidth of the narrow resonances is about $4 \mathrm{~cm}^{-1}$ : though the material is spatially strongly inhomogeneous, both homogeneous broadening due to phonon decay and inhomogeneous broadening due to random crystallite sizes still allow for sharp resonances. We note that the transmission study of powder samples ${ }^{14}$ delivers the same phonon structure, with minima where the reflection spectrum has maxima; however, the halfwidth of the resonances is (by about a factor of 4) larger than for the reflection spectrum.

The reflectance spectrum (Fig. 1b) for the single phase sample on the other hand is smooth and shows only weak phonon structure. The form of the spectrum for the single phase sample is dominated by high-frequency electronic conduction. For an analysis of the reflectance spectrum for the single phase sample at $10 \mathrm{~K}$ we have drawn in Fig. 1b (dotted) a reflectance curve calculated by Genzel et al. ${ }^{8}$ In this analysis electronic conductivity is taken into account in addition to phonon resonances; a reflectance step (in the $10 \mathrm{~K}$ curve) is consistent with a superconducting energy gap at $220 \mathrm{~cm}^{-1} .8$

For the mixed phase sample an influence of electronic effects on the reflectance is clearly seen at low frequencies: 
The reflectance increases towards low frequencies. For a description of the increase of the reflectance at low frequencies we first performed an analysis assuming a Drude term as an additional contribution to the dielectric function. However, such an analysis leads to a plasma frequency that is an order of magnitude smaller and a scattering rate that is two orders of magnitude smaller than for the single phase sample ${ }^{8}$ and is therefore not realistic. Furthermore, due to a large contribution to the dielectric function, the strong minimum in the reflection curve at $118 \mathrm{~cm}^{-1}$ (Fig. 1a) and other minima were smeared out.

We found that the reflectance curve (Fig.1a) for the mixed phase superconductor was very similar to a reflectance curve for a single phase $\mathrm{Y}_{2} \mathrm{BaCuO}_{5}$ insulating ceramic material (that has green colour). We write therefore

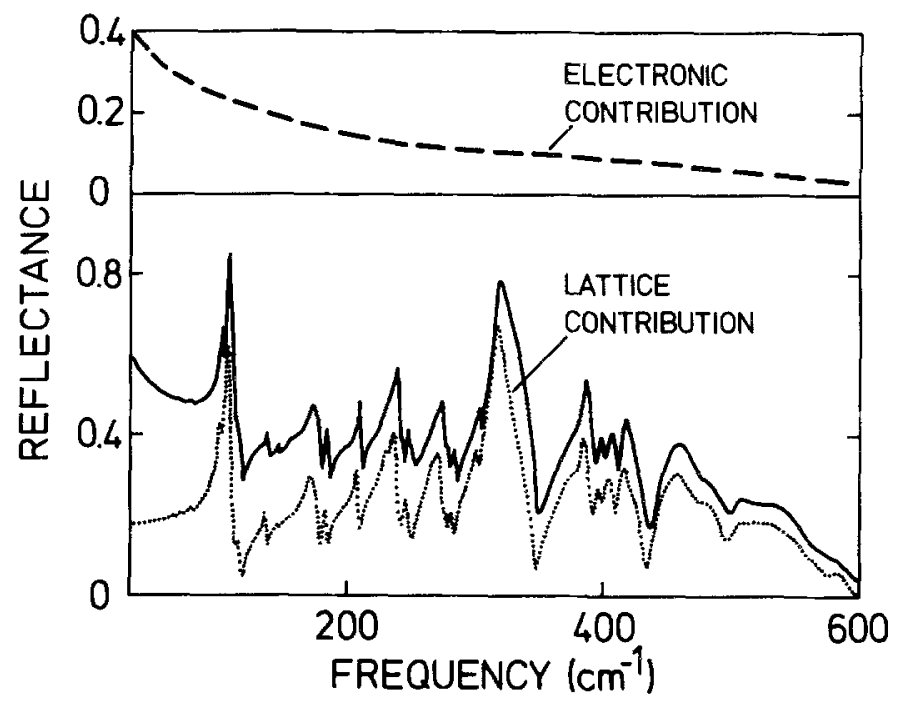

Figure 2. Reflectance of the mixed phase superconductor $\mathrm{Y}_{1.2} \mathrm{Ba}_{0.8} \mathrm{CuO}_{4-\mathrm{y}}$. The experimental curves can be described by lattice reflection (dotted) and additional reflection (upper curve) at a mesh-like structure of conducting material. 
the reflectance of the mixed phase sample as a sum $R=\frac{4}{5} R_{i}+\Delta R$ where $R_{i}$ is the reflectance of the insulating material and $\triangle R$ an additional reflectance caused by the conducting material embedded in insulating material. The dotted curve in Fig. 2 shows the reflectance $\frac{4}{5} R_{i}$ that follows from the experimental reflectance for the insulating material that has a low-frequency dielectric constant $\varepsilon \simeq 8$ and a high-frequency dielectric constant $\varepsilon_{\infty} \simeq 3$. From the difference $R-\frac{4}{5} R_{i}$ we find an additional reflectance shown in the upper part of Fig. 2. We attribute the additional reflectance to a contribution from a mesh-like structure consisting of conducting filaments. By comparison with reflectance curves that are known for metallic mesh ${ }^{15}$ we find a typical distance of conducting filaments of the order of few $10^{-3} \mathrm{~cm}$. This is consistent with visual inspection of our sample.

The transition from normal conductivity to superconductivity in the filaments would only lead to a small change of the reflectance, which is in accordance with our experiment (Fig. 1a).

In conclusion, the far infrared reflectance behavior of a mixed phase superconductor $\mathrm{Y}_{1.2} \mathrm{Ba}_{0.8} \mathrm{CuO}_{4-Y}$ is consistent with lattice reflection due to nonconducting material and additional reflectance due to conducting material of mesh-like structure.

Acknowledgement - We would like to thank L. Genzel for stimulating discussions, for information about unpublished studies and we thank W. König (Stuttgart) for performing the far infrared reflection measurements. The work has been supported by the Bundesministerium für Forschung und Technologie.

\section{References}

1. J.G.Bednorz and K.A.Müller, Z. Phys. B 64, 189 (1986).

2. M.K.Wu, J.Ashburn, C.J.Torng, P.H.Hor, R.L.Meng, L.Gao, Z.J.Huang, Y.Q.Wang and C.W.Chu, Phys. Rev. Lett. 58, 908 (1987). 
3. P.E.Sulewski, A.J.Sievers, S.E.Russek, H.D.Hallen, D.K.Lathrop and R.A.Buhrman, Phys. Rev. B 35. 5330 (1987).

4. Z.Schlesinger, R.L.Greene, J.G.Bednorz and K.A.Müller, Phys. Rev. B 35, 5334 (1987).

5. U.Walter, M.S.Sherwin, A.Stacy, P.L.Richards and A.Zettl, Phys. Rev. B 35, 5327 (1987).

6. P.E.Sulewski, A.J.Sievers, R.A.Buhrman, J.M.Tarascon, L.H. Greene and W.A.Curtin, to be published.

7. D.A.Bonn, J.E.Greedan, C.V.Stager, T. Timusk, M.G.Doss, S.L.Herr, K. Kamaras and D.B.Tanner, Phys. Rev. Lett. 58, 2249 (1987).

8. L. Genzel, A.Wittlin, J.Kuhl, Hj.Mattausch, W.Bauhofer and A.Simon. Sol.St.Comm. 63, 843 (1987).

9. M.Cardona, L.Genzel, R.Liu, A.Wittlin, Hj.Mattausch, F. Garcia-Alvaredo and E.Garcia-Gonzales, to be published in Sol.St. Comm.

10. S.Onari, M.lioka, K.Ohshima, T.Arai and T.Sakudo, Jap. J.Appl.Phys. 26, L1052 (1987).

11. G.A.Thomas, H.K.Ng, A.J.Millis, R.N.Bhatt, R.J. Cava, E.A.Rietman, D.W.Johnson,Jr., G.P. Espriosa and J.M. Vandenberg, Phys.Rev. B36, 846 (1987).

12. S.B.Quadri, L.E.Toth, M.Osofsky, S.Lawrence, D.U.Gubser and S.A.Wolf, Phys. Rev. B 35. 7235 (1987).

13. R.J.Hemley and H.K.Mao, Phys. Rev. Lett. 58, 2340 (1987).

14. T.H.H.Vuong, D.C.Tsui, V.J.Goldman, P.H.Hor, R.L.Meng and C.W.Chu, Sol.St.Comm. 63, 525 (1987).

15. K.F. Renk and L. Genzel, Appl.Opt. 1, 646 (1962). 